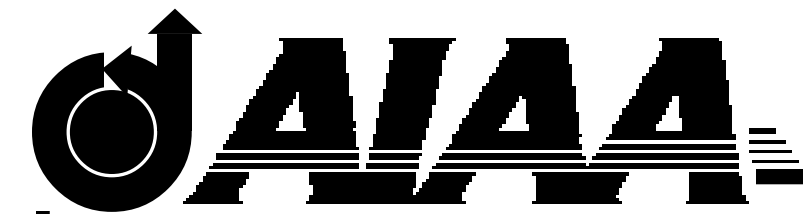

\title{
AIAA 95-3619 \\ Defining Support Requirements \\ During Conceptual Design of \\ Reusable Launch Vehicles
}

W. D. Morris

N. H. White

W. T. Davis

NASA Langley Research Center Hampton, Virginia

\section{Dr. C. E. Ebeling}

University of Dayton

Dayton, Ohio

\section{AIAA 1995 Space Programs and Technologies Conference September 26-28, 1995/Huntsville, AL}




\title{
DEFINING SUPPORT REQUIREMENTS DURING CONCEPTUAL DESIGN OF REUSABLE LAUNCH VEHICLES
}

\author{
W. D. Morris*, N. H. White**, W. T. Davis** \\ NASA Langley Research Center \\ Hampton, Virginia \\ Dr. C. E. Ebeling † \\ University of Dayton \\ Dayton, Ohio
}

\begin{abstract}
Current methods for defining the operational support requirements of new systems are data intensive and require significant design information. Methods are being developed to aid in the analysis process of defining support requirements for new launch vehicles during their conceptual design phase that work with the level of information available during this phase. These methods will provide support assessments based on the vehicle design and the operating scenarios. The results can be used both to define expected support requirements for new launch vehicle designs and to help evaluate the benefits of using new technologies. This paper describes the models, their current status, and provides examples of their use.
\end{abstract}

\section{Nomenclature}

$\begin{array}{ll}\text { BCS } & \text { Baseline Comparison System } \\ \text { DOD } & \text { Department of Defense } \\ \text { DSE } & \text { Depot Support Equipment } \\ \text { ECLS } & \text { Environmental Control and Life Support } \\ \text { GPOT } & \text { Ground Power On Time, hours } \\ \text { IEP } & \text { Induced Environmental Protection } \\ \text { IOC } & \text { Initial Operating Condition } \\ \text { KSC } & \text { Kennedy Space Center } \\ \text { L } & \text { Length } \\ \text { LaRC } & \text { Langley Research Center } \\ \text { LRU } & \text { Line Replaceable Unit } \\ \text { MTBM } & \text { mean time between maintenance, hours } \\ \text { MTBR } & \text { mean time between removal, hours } \\ \text { MTTR } & \text { mean time to repair, hours }\end{array}$

*Aerospace Technologist, Space Systems and Concepts Division, Member AIAA.

**Aerospace Technologist, Space Systems and Concepts Division.

$\dagger$ Associate Professor, School of Engineeering.

Copyright $@ 1995$ American Institute of Aeronautics and Astronautics, Inc. No copyright is asserted in the United States under Title 17, U.S. Code. The U.S. Government has a royalty-free license to exercise all rights under the copyright claimed herein for Governmental purposes. All other rights are reserved by the copyright owner.

$\begin{array}{ll}\text { NSLD } & \text { National Shuttle Logistics Depot } \\ \text { O\&S } & \text { Operations and Support } \\ \text { OMI } & \text { Operations Maintenance Instructions } \\ \text { OMRSD } & \begin{array}{l}\text { Operations and Maintenance Requirements and } \\ \text { Specification Document }\end{array} \\ & \text { Personnel Launch System } \\ \text { PLS } & \text { Purge Vent and Drain } \\ \text { PVD } & \text { Reliability and Maintainability } \\ \text { R\&M } & \text { Reaction Control System } \\ \text { RCS } & \text { Single Stage Vehicle } \\ \text { SSV } & \text { Thermal Control System } \\ \text { TCS } & \text { Workhours/Maintenance Action } \\ \text { WH/MA } & \text { Wingspan } \\ \text { WS } & \text { Weight } \\ \text { Wt. } & \text { manhours } \\ \text { workhours } & \end{array}$

\section{Introduction}

Methods have been developed over the last 30 to 40 years as a part of the systems engineering process, by both the military and commercial analyst to define the support requirements for new aircraft concepts. ${ }^{1-3}$ Generally these methods have been applied during development phases where the system is fairly well defined. As such, the methods have frequently been data intensive and required an extended level of definition in order to be applied. In conceptual design studies, such as those performed at the NASA Langley Research Center (LaRC), application of these same methods to launch vehicle designs has been limited both by the reduced level of definition available and by the lack of applicable historical data for reusable space vehicles. Conceptual designs, by their nature, provide very limited vehicle definition. In order to define the support requirements and to discriminate among new technology choices for these systems, it has been necessary to develop new analysis methods. These methods must be capable of working with a limited level of concept definition to define the support required consistent with both the design and operational concepts. 
Early attempts to define support for conceptual launch vehicles focused on the use of discrete event simulation modeling. ${ }^{4-6}$ Although useful in giving general insight to support requirements, the models had to be based on assumed parametric values such as turnaround time, manpower, number of facilities, etc. Historically defined support requirements were generally only available at highly aggregated levels. This level lacked the fidelity necessary to evaluate the effects of introducing new technologies. Additional data was obtained in a study specifically designed to aid in process definition, and define manpower and task times for launch operations. ${ }^{7}$ While this information aided simulation modeling there still lacked a direct connection between the design and its support requirements. This linkage to the design is usually through the reliability and maintainability $(\mathrm{R} \& \mathrm{M})$ requirements. In order to establish this link in the absence of R\&M data from launch vehicles, the approach was to define support based on comparability to aircraft system requirements. ${ }^{8}$

Aircraft data were used to formulate an analysis tool based on parametric estimating relationships. ${ }^{9}$ This method builds on one developed by Weber ${ }^{10}$ for analyzing space system designs based on aircraft data. As Shuttle data became available in the post Challenger time period, a Martin Marietta ${ }^{11}$ study was used to define R\&M data from the Shuttle program that was comparable to the aircraft data used by the analysis model. This study provided Shuttle data comparable to the aircraft reliability histories, but required major assumptions to develop maintainability data. A more recent study has confirmed that the maintenance data is not available from currently existing Shuttle electronic databases. ${ }^{12}$

The concept of defining support in terms of vehicle parameters was extended to the study of logistics by also determining parameters that characterize the logistics support environment. Logistics models were developed by Rockwell as a part of the Personnel Launch System (PLS) studies ${ }^{13}$ and were later expanded in a study that attempted to define the parameters that characterize the Shuttle and aircraft support environments. ${ }^{14}$ An earlier attempt to combine these approaches into a unified analysis for the HL-20 is captured in reference 15. This paper will describe several methods under development at LaRC, give their current status, and provide an example of their use in defining support requirements for a single-stage-to-orbit vehicle.

\section{Models and Analysis Methods}

Support requirements of new systems encompass both the ground and flight operations. This includes not only the direct cost for organizational level maintenance and servicing, but also the logistical support which includes the facilities, supplies, transportation, training, documentation, depot maintenance and management. Comparability analysis in which the support requirements of future systems are defined based on similarities to known support requirements of existing systems formed the basis of the analysis method. Three criteria were established for the analysis tools that are being developed: 1) they must work with the limited type of data available in conceptual design studies, 2) they must link the design and its operational performance to the operations and support (O\&S) environment, and 3) where possible the methods need to be based on historical data. The methods being developed have emerged to form a set of analysis tools. They are an R\&M model, a logistics model, and the use of discrete event simulation modeling. Each can be used as a standalone tool, or combined with the others to provide a more complete analysis.

\section{R\&M Model}

The R\&M model addresses the definition of reasonable expectations for turnaround times and manpower requirements of conceptual vehicles. It is predicated on the assumption that these requirements should be based on the maintenance actions generated by each mission and the maintenance policy that is chosen to return the vehicle to flight worthiness. The model provides the critical link between the operating scenario and the vehicle design. It is based on the assumption of comparability to either aircraft or Shuttle subsystem support requirements (Figure 1). R\&M data from both aircraft (37 different aircraft over a 2 year period of operation) and Shuttle support histories (16 post Challenger flights) were used in developing the model.

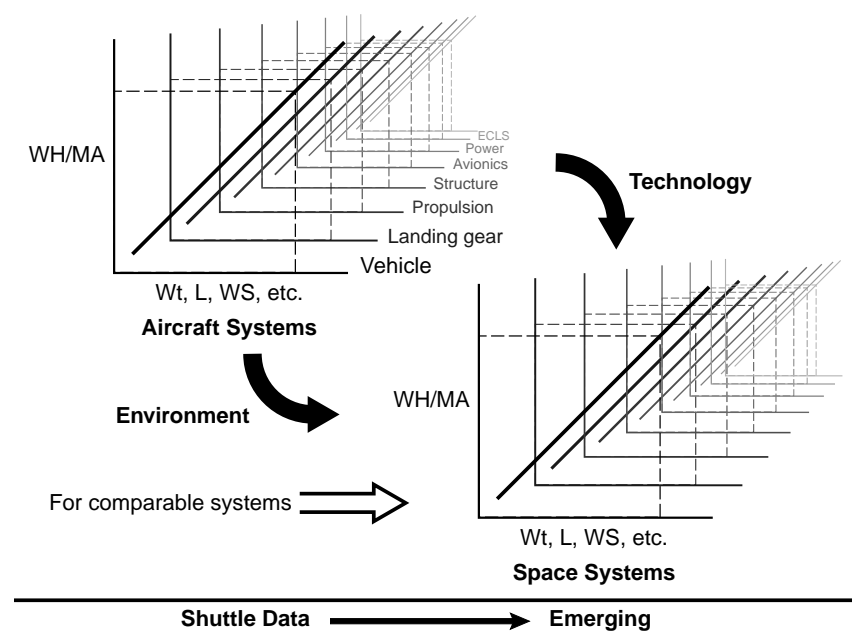

Figure 1. Reliability and maintainability model. 
The number of maintenance actions and the number of maintenance workhours required for each subsystem are estimated based on the user's choice of comparability to either aircraft or Shuttle reliability and maintainability characteristics. These are primarily the mean time between maintenance (MTBM), mean time to repair (MTTR), a technology growth factor, and the critical failure rate (Table 1). The MTBM is a measure of the system's operational reliability and is used to indicate the frequency that maintenance must be performed on a system. The MTTR is a measure of the time required for properly skilled crew with all of the necessary resources to return a system to operating status, and is a measure of the systems inherent maintainability. A technology factor was developed by observing the improvement in MTBM characteristics over a period of years, then interpreting that change as a rate of growth that can be applied to similar subsystems. This is used to project an expected improvement in the database technologies to the time period of the study. The critical failure rate is based on the percentage of maintenance actions that have resulted in aborts out of the total number of maintenance actions for each subsystem based on aircraft data and is used to define the phased reliability of the system.

Table 1. Operations and Support Drivers

\begin{tabular}{lc}
\hline \hline Maintenance Actions (R\&M) & Maintenance Policy \\
\hline - MTBM & $\cdot$ Ratio scheduled/ \\
- MTTR & unscheduled maintenance \\
- Technology growth factor & $\bullet$ Crew size \\
- Critical failure rate & $\cdot$ Ground power on time (GPOT) \\
\hline \hline
\end{tabular}

Maintenance policies are input through the choices of parameters that reflect those characteristics of either Shuttle or aircraft maintenance support policies or the user can create his own policy. The primary parameters used to define the maintenance concept are the ratio of scheduled to unscheduled maintenance, the crew size required to do the hands-on labor and the power-on time required for ground servicing (GPOT). The amount of unscheduled maintenance performed on aircraft has been observed to be about twice the scheduled maintenance required (for this size vehicle). This characteristic is used to define an aircraft maintenance concept in which the amount of scheduled maintenance reflects the maturity of a system which has allowed the amount of preventive maintenance to be balanced against the risk of failed systems. Developing the same characteristic for Shuttle, unscheduled maintenance is about $20 \%$ of the scheduled maintenance. This is consistent with the Shuttle maintenance concept which requires extensive inspection and testing between flights in order to ensure successful system operation. The size of the crew required to support maintenance on each subsystem reflects the number of unique skills required for that technology. For aircraft, this normally involves a crew chief and one or two technicians with specialized skills required for the task. For Shuttle, the crews frequently are made up of a test conductor, a systems, quality, and safety engineer, and a technician. This crew size and makeup reflects a maintenance concept driven by complex vehicle and ground systems designs, and frequently requires engineering effort to support the maintenance activities. As the system matures, and failure modes appear frequently enough that they become well documented, the need for unique solutions from engineering support should lessen as repair methods become 'standardized.' This should substantially reduce the number of maintenance activities and reduce the need for large crew sizes and engineering support. The current maintenance concept used on Shuttle requires extensive periods of time when the power is on for the flight systems while they are being tested. This increased time of operation and increased exposure to induced damage has a direct effect on the amount of maintenance required.

The R\&M model input is matched to the level of definition available from the weights and sizing model. The weights and sizing model is used to develop the vehicle dimensions and subsystem weights based on the vehicle performance requirements. The R\&M model input requires vehicle definition in terms of overall dimensions, weight, and technology if available. Individual subsystem weights and other characteristics can be used to provide better definition. Mission length and space environmental differences are accounted for when using the aircraft data, however the use of Shuttle data requires adjustment of the R\&M values by the user to account for these differences. The user must adjust the Shuttle derived values to account for the physical characteristics which are different from the Shuttle's system and to account for differences in the mission environments. The output can then be used to define the turnaround and manpower requirements based on the system design and choice of maintenance concept (Figure 2).

There are many different ways that R\&M model can be used for analysis, but a typical example of how it is used for conceptual studies is as follows. Once a case is built based on the vehicle description, weights and flight rate objectives, runs are then made with the model in order to define the $R \& M$ characteristics of the concept for two bracketing conditions. First it is defined based on comparability to Shuttle R\&M characteristics and support concepts. Next it is de- 


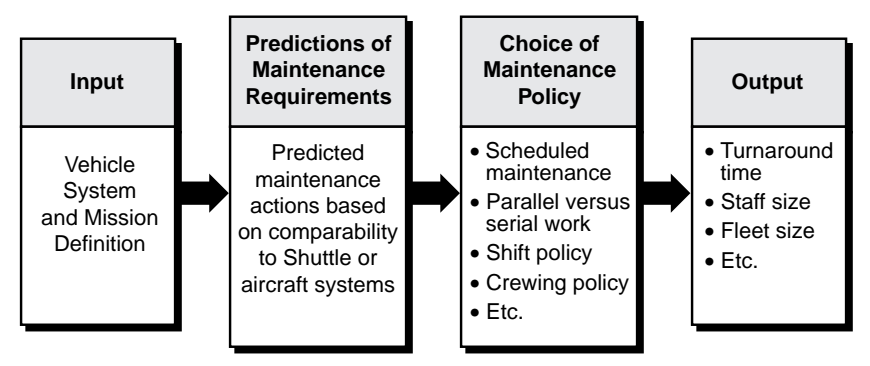

Figure 2. Analysis process for R\&M model.

fined based on comparability to aircraft characteristics and support concepts. For those systems for which there are no comparable aircraft systems, assumptions of improvements are made based on the Shuttle values. This creates a range of R\&M parameters between the currently demonstrated capability of Shuttle (Shuttle values), and a set of values characteristic of aircraft. In general, the Shuttle R\&M values represent the current capability and the aircraft values the potential goals for new launch vehicles.

Then a Baseline Comparison System (BCS) (Figure 3) is developed based on either improvements in the Shuttle based reference values (things whose trends reduce the overall support requirements), or values based on characteristics that are within the capability demonstrated by the aircraft reference system. The BCS model is simply a composite of the R\&M parameters from existing systems which are used to represent the characteristics of the new concept. This model has traceability back to known systems. For Shuttle this means specific hardware, but for aircraft values it cannot lead back to a specific aircraft, but to the parametric equation representing the aircraft. When the desired operating characteristics are achieved, the resulting subsystem R\&M characteristics can be used as initial $R \& M$ requirements allocations.

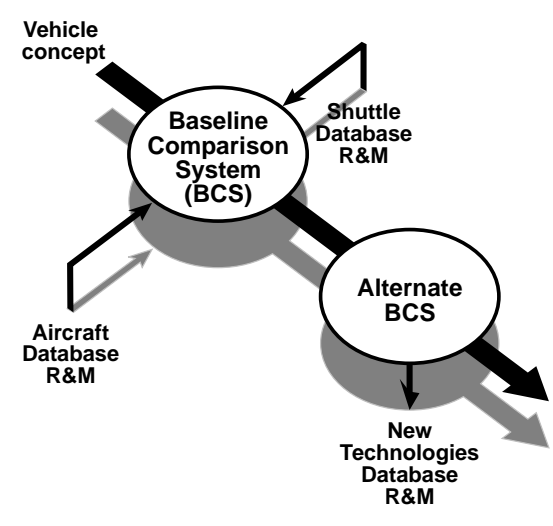

Figure 3. Design definition process.

\section{Logistics Model}

The logistics model is based on cost elements typically associated with the support of any system: maintenance, support equipment, training, documentation, supplies, transportation, and management (Figure 4). Maintenance support is a function of the number of maintenance actions required, the time required to repair, the touch manpower required, the frequency and cost of replacement parts, and the flight rate for the fleet of vehicles. Many of these input values are output from the R\&M model, although the logistics model has supporting algorithms for these if not otherwise available. The training costs are a function of the number of courses, the cost required to develop and administer the training as well as the number of personnel and the time required to take the training. These are a function of both the design and the maintenance policy. At this time computer based training is not accounted for by the model.

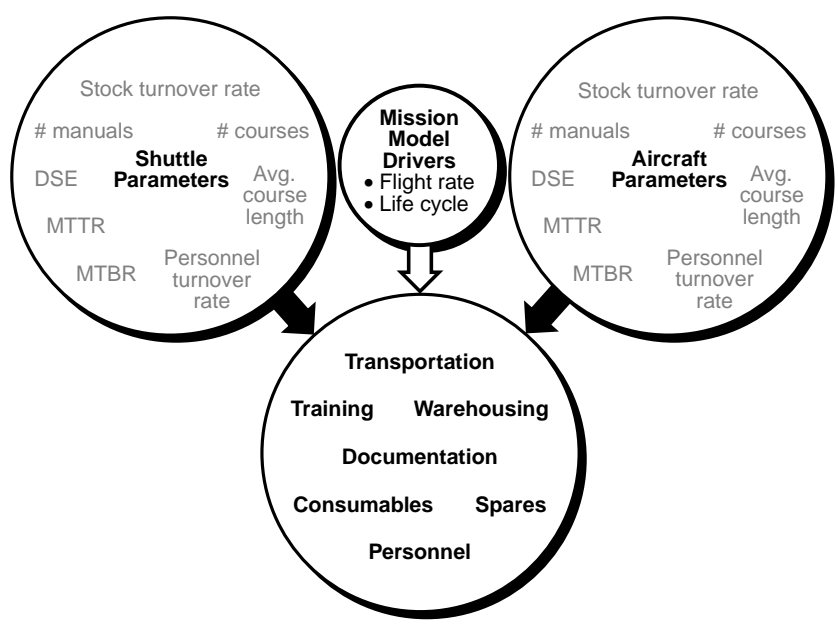

Figure 4. Logistics modeling approach.

The documentation is primarily the development, publication, and updating of the maintenance manuals. These are driven by the number of systems on the vehicle, the number of unique reparable line replaceable units (LRU), and the number of pages required in the manuals. At this time the model does not account for electronic documentation. The supply support includes the cost of buying, storing and managing spares and consumables. The spares cost are a function of the total number of LRUs on a vehicle, removal and condemnation rates, the time required for the repair cycle on these parts, the flight rate, and the sparing policy. The management cost is a function of the cost to stock and maintain the spares inventory. The consumables cost is primarily a function of the flight rate. The transportation cost includes 
both transport of the vehicle to the launch site and the cost of transporting spares to and from the depot site. The cost of support equipment is currently based on Shuttle support equipment cost for both the aircraft and Shuttle environments. The cost of equipment is assumed to be proportional to the Shuttle's cost based on vehicle size, turnaround time, and flight rate. Support environment cost for aircraft are arbitrarily assumed to be half that of Shuttle.

This set of algorithms were written such that selected independent parameters could be used to characterize the support equipment as either based on Shuttle logistics support or military aircraft logistics support. Of over 50 non-vehicle specific parameters identified, only a few have been defined with values that uniquely differ between these two environments. Primary among them are the repair times, size of repair crews, training times, amount of documentation, time required for ground processing, and the amount of scheduled maintenance required (both organizational and depot level).

Inputs required are vehicle description, mission, and the choice of support type typified by either the aircraft or Shuttlelike environments. This work was predicated on the assumption that these differing values would characterize the two different approaches to logistics support. It was also assumed that going from Shuttle to aircraft type support would represent an improvement in efficiency and effectiveness of the support. These algorithms were developed under contract by Rockwell. ${ }^{14}$ The primary cost drivers and their relationships were based on their experience in logistics support. The primary data sources used were from the National Shuttle Logistics Depot (NSLD) for Shuttle, and Department of Defense (DOD) for aircraft.

The model typically uses the output from the R\&M model as input. These are primarily the turnaround time, the handson vehicle level crew size and the fleet size. Program input requires definition of the year on which the technology is based and the planned operating life. The model also requires inputs of overall vehicle weights, mission description phase times and propellant types. In addition, the support and operating scenario is described in terms of launch and landing sites, manufacturing site, and depot location (for determining transportation cost). The model uses this information to estimate both the non-recurring and recurring cost to establish and operate the system over its life cycle. The independent parameters used by the model are chosen to describe the support environment as either similar to aircraft or Shuttle type support environments.
Fundamental to the model is the assumption that the organizational support requirements are driven by the unscheduled maintenance requirements of the design. Both the time and personnel required to return it to flight status are also driven by the maintenance concept that has been assumed for this system in the R\&M model. In addition the number of systems and subsystems that must be supported are drivers in the logistic support. Both the number of removals and the repair cycle time and personnel are primary drivers in the depot level of logistics support.

\section{Simulation Model}

Discrete event simulation modeling has been a standard tool for evaluating operational scenarios. It can be used for analysis of a single flow or simulating the operational environment over the life cycle of the system (Figure 5). The models can be used to examine the delays and conflicts that occur when demands are placed on limited resources. This provides the opportunity to better define the level of resources needed when the concept is used in this environment. It also accounts for failures and the variances that will occur in the operating scenario so that the level of resources can reflect the probability of these occurrences. Model results are scenario dependent and rely on input definitions of flight rate requirements and task durations. The operating and maintenance scenarios place demands on the resource requirements as the simulation progresses, defining the level of support required for the scenario. Output is in the form of flight rate capabilities, resource requirements, and facility utilizations. This tool is used to assess the impact of scenario variations on support requirements.

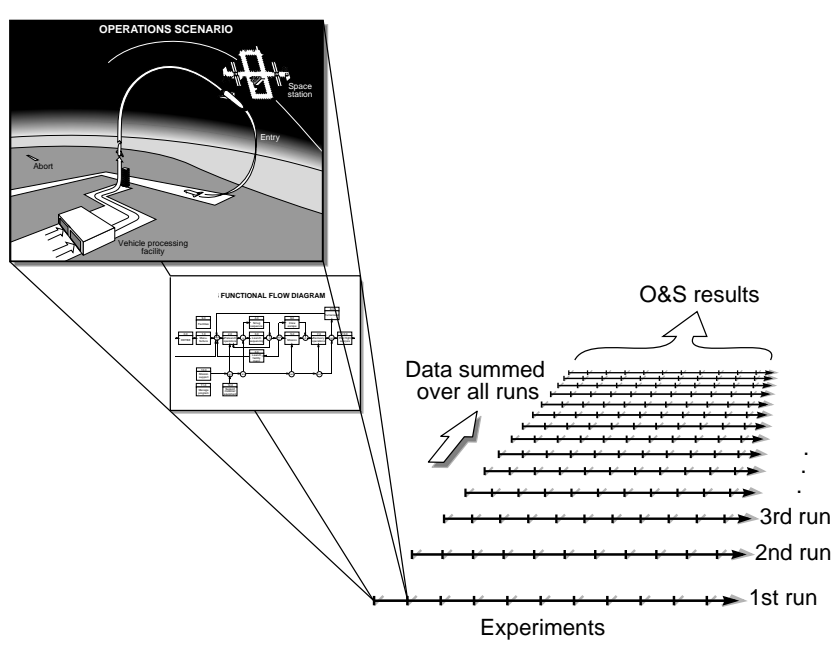

Figure 5. Simulation modeling for operations. 


\section{Results \& Discussion}

A Single Stage launch Vehicle (SSV) supporting low earth orbit missions was used to illustrate the models. The vehicle description provided by the design team (Figure 6) is similar to that described in reference 16 . The launch vehicle, which did not require a flight crew, was designed to deliver up to 25,000 pounds to orbit, stay up to 7 days and return. Using advanced technologies, 7 engines and cryogenic fuels, the vehicle had a dry weight of 200,300 pounds (margin included), a wingspan of 93 feet and an overall length of 186 feet.

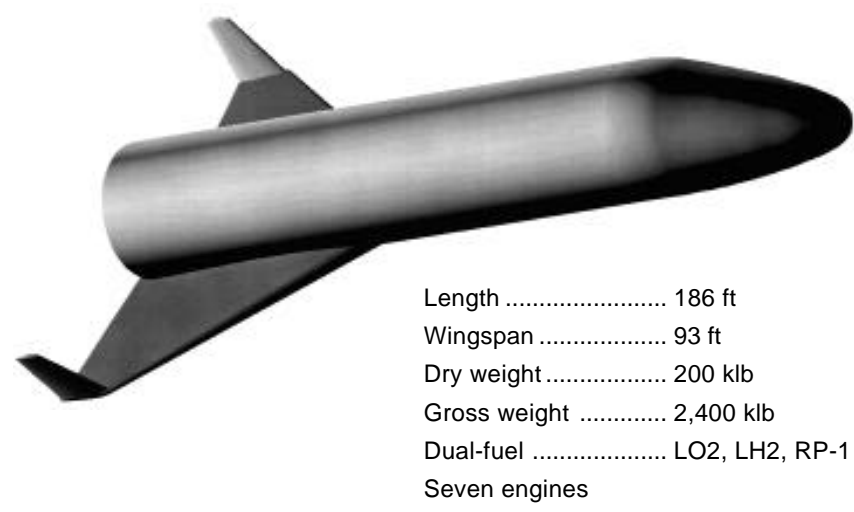

Figure 6. Single-stage vehicle (SSV) concept.

The operating scenario used for this example is shown in Figure 7. An initial operating capability (IOC) of 2007 was used. Thirty missions were flown a year with a mission length of 7 days. The support concept was for a two-level maintenance system with all vehicle level unscheduled and scheduled maintenance being performed in the processing bay. The payload was also integrated within this bay. The processed vehicle with payload installed is transported to the launch pad where it is erected using a strong back and attached to the launch pad. Launch operations are assumed to take 12 hours. Support and launch are from Kennedy Space Center (KSC) and nominal return is to KSC. The depot level support facility is to be located on site. Launch vehicle manufacturing and support spares are assumed from a west coast site.

All three models were exercised to obtain results for this concept. First a top level estimate of the support was developed using the R\&M model. Output from this was then used as input to both the logistics and the simulation model to further define the overall operations and support requirements.

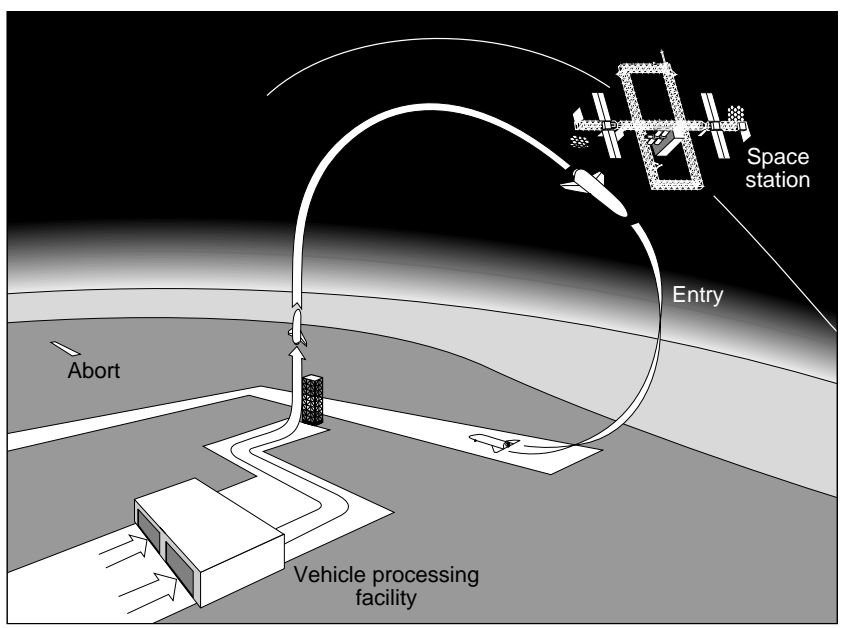

Figure 7. Operating scenario.

\section{R\&M Model Results}

The predicted maintenance burden per flight for the SSV to meet flight rate goals is shown in Figure 8 for different sets of R\&M and maintenance concepts (as defined in Table 1). The figure defines an area of feasible design space for operational concepts. The horizontal axis represents changes in the $\mathrm{R} \& \mathrm{M}$ characteristics varying from aircraft-like to Shuttle-like values. The axis into the paper represents the effect of varying the maintenance concept characteristics from aircraft-like to Shuttle-like. The vertical axis defines the associated maintenance burden in workhours that results from using the R\&M and Maintenance concept characteristics chosen for each concept. For this figure, the 23 different subsystems that are used to define the vehicle have been grouped into the structural, tanks, thermal protection, propulsion, power, avionics, environmental control, mechanical and auxiliary systems.

Figure 8a illustrates the most optimistic case where both aircraft R\&M values (MTBM, MTTR, Crew Size, etc.) and maintenance concepts (scheduled to unscheduled ratio, GPOT, crew size, etc.) were used wherever possible. Parameters were chosen for those where no aircraft data existed based on aggressively assumed improvements to Shuttle R\&M characteristics (Tiles, TCS, PVD, and Fuel Cell assumed a $10,5,5$, and 3 fold increase in reliability respectively.). If aircraft-like support can be achieved, the touch labor maintenance burden expected for this support concept is 2,000 workhours. A manpower leveling was done on selected subsystems that appeared to be driving the turnaround times to achieve a vehicle turn time of approximately 10 workdays (based on working 1 shift/day, 5 days/week). This 


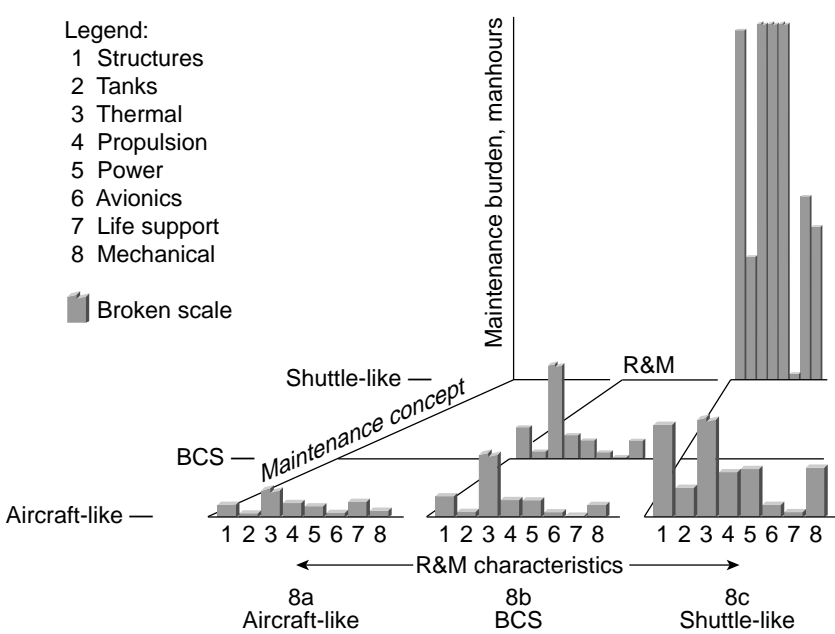

Figure 8. Maintenance burden as a function of $R \& M$ and maintenance concept characteristics.

resulted in a manpower requirement of 136 hands-on support personnel for performing productive work for a fleet size of 3 vehicles.

Figure $8 \mathrm{c}$ illustrates the maintenance burden per flight that would result if subsystem R\&M characteristics from Shuttle and an aircraft-like maintenance concept are used, based on assumed technology improvements that could be expected from current technologies by the year 2007. The total maintenance burden expected for this support concept is 29,000 workhours. A manpower leveling was done on selected subsystems that appeared to be driving the turnaround times to achieve a vehicle turn time of approximately 12 days. This resulted in a manpower requirement of 680 hands on support personnel for performing productive work. The upper part of figure $8 \mathrm{c}$ illustrates the maintenance burden when the Shuttle maintenance concept is used (high proportion of scheduled to unscheduled maintenance, larger crew sizes, more ground power on time for processing, etc.) This total maintenance burden is 113,000 workhours. A manpower leveling was done on selected subsystems that appeared to be driving the turnaround times to again achieve a vehicle turn time of approximately 48 days. This resulted in a manpower requirement of 940 hands on support personnel for performing productive work for a fleet size of 7 vehicles to achieve 30 flights per year.

Figure $8 \mathrm{~b}$ then represents the baseline comparison system (BCS) for this example. It is based on achieving MTBM and MTTR values that are greater than the aircraft values by $50 \%$ of the difference between that achieved by aircraft and Shuttle, and crew sizes that require one person per crew in addition to that required in aircraft maintenance (Table 2). In no cases were characteristics used that would require more support than Shuttle values. This results in a maintenance burden of 4,400 workhours and a support crew of 180 to achieve 12 day turn time. To account for a more Shuttle-like maintenance concept that provides a higher level of inspection, a scheduled maintenance ratio was assumed double that observed in aircraft to account for the increased work required. Ground operating times were also doubled from the aircraft concept to account for increased inspection times (BCS maintenance concept). This results in a maintenance

Table 2. R\&M Characteristics

\begin{tabular}{lccc}
\hline \hline & Aircraft & BCS & Shuttle \\
\hline Wing Group & & & \\
MTBM & 9.12 & 6.78 & 4.45 \\
MTTR & 3.71 & 4.23 & 4.75 \\
Crew size & 1.85 & 2.85 & 4.5 \\
Tanks-LOX & & & \\
MTBM & 16.65 & 16.65 & 16.65 \\
MTTR & 2.59 & 4.03 & 5.47 \\
Crew size & 1.85 & 2.85 & 4.5 \\
Propulsion-RCS & & & \\
MTBM & 20.15 & 16.80 & 13.45 \\
MTTR & 2.39 & 5.15 & 7.92 \\
Crew size & 2.43 & 3.43 & 9 \\
IEP-TCS & & & \\
MTBM & 24.95 & 14.97 & 4.99 \\
MTTR & 6.6 & 6.6 & 6.6 \\
Crew size & 4.5 & 4.5 & 4.5 \\
IEP-PVD & & & $\cdot$ \\
MTBM & 384.45 & 230.66 & 76.87 \\
. & $\cdot$ & $\cdot$ & $\cdot$ \\
· &. & $\cdot$ & $\cdot$ \\
\hline
\end{tabular}

Table 3. Support Characteristics

\begin{tabular}{lccccc}
\hline \hline & Aircraft & BCS & BCS & Shuttle & Shuttle \\
& R\&M & R\&M & R\&M & R\&M & R\&M \\
& MC* $^{*}$ & MC & MC & MC & MC \\
\hline Workhours & 2000 & 4400 & 4900 & 29000 & 113000 \\
Turn time, days & 10 & 12 & 12 & 12 & 48 \\
Manpower & 136 & 180 & 210 & 680 & 940 \\
Fleet size & 3 & 3 & 3 & 3 & 7 \\
\hline \hline
\end{tabular}

${ }^{*} \mathrm{MC}$ - Maintenance concept 
burden of 4,900 workhours and a support crew of 210 to achieve 12 day turn time for a fleet size of 3 . These results are summarized in Table 3.

\section{Logistics Model Results}

The Logistics model was then used for this same vehicle to estimate the effects of different support concepts on the relative costs of each, for both the non-recurring and recurring costs. The turnaround times, fleet size, and support crew size were taken from the output of the R\&M model. An operating life cycle of 25 years was assumed. The distribution of operating costs for the eight logistics cost areas are illustrated in figure 9 for the three different support environments: that typical of Shuttle, typical of aircraft, and the environment chosen for this vehicle concept.

The results shown for the chosen support environment is bounded by the results that would be achieved if the parameters characteristic of either the Shuttle or aircraft support environments had been chosen for this vehicle. Figure 9a illustrates the effects on the non-recurring cost required to acquire material and train the support personnel (touch only) prior to the initial operating capability. Supplies, support equipment and documentation are the dominant cost elements for the BCS concept. This might be expected as it captures the initial cost of laying in supplies, purchase of the support equipment, and developing the maintenance manuals. Initial support equipment cost are higher for the as-Shuttle case. This reflects the additional equipment that is required because of the longer processing times for this maintenance policy. The Shuttle's documentation costs are significantly higher than aircraft because of the need for Operations and Maintenance Requirements and Specification Documents (OMRSD) in addition to the Operations Maintenance Instructions (OMI) which are more equivalent to the typical maintenance manual The BCS support assumes a $65 \%$ reduction in OMRSD documentation needs. The supplies cost are essentially the same for all concepts because the same sparing policy was used in all three cases. An overall $44 \%$ reduction from Shuttle-like support cost would be achieved with the assumptions used for the non recurring logistics support.

The same logistics cost elements are drivers of the annual recurring or operating cost of the system as shown in figure 9b. Using Shuttle logistics support parameters, maintenance cost is a driver. For the BCS logistics support concept the maintenance policy was more aircraft like and reflects reductions in maintenance actions and ground processing times from that used in Shuttle processing. These

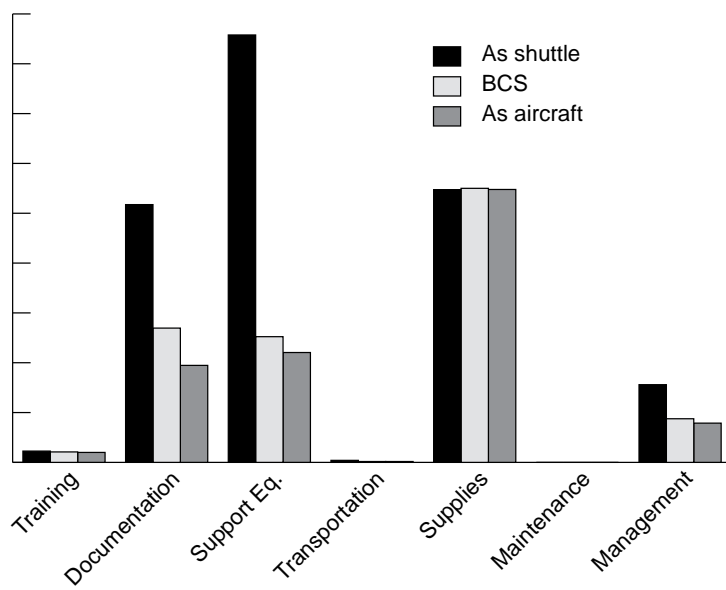

a. Non-recurring.

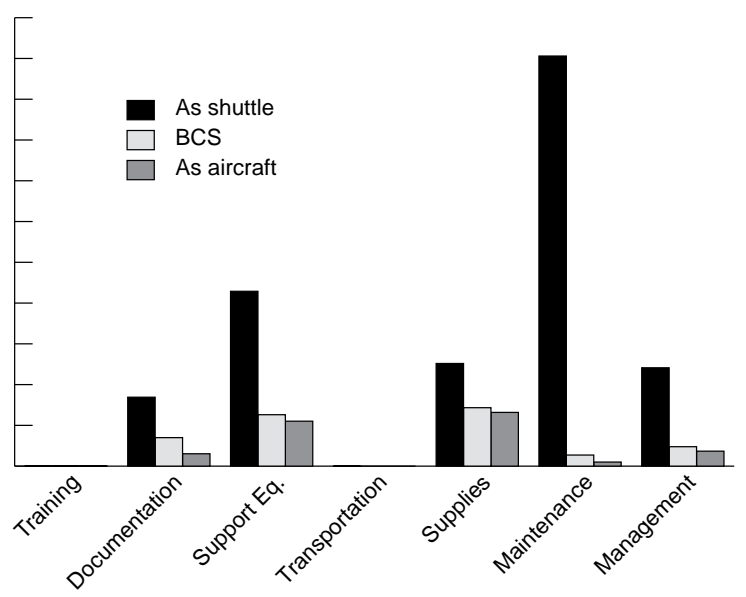

b. Annualrecurring.

Figure 9. Logistics cost elements.

reductions result in dramatically lower cost due to the smaller maintenance burden. The reduced number of maintenance actions has also reduced the number of removals and the supplies cost. The support equipment cost are a percentage of the non-recurring cost to account for replacement and repair of the equipment. An overall 80\% reduction from Shuttle type operations cost would be achieved with the assumptions used for the recurring logistics support.

\section{Simulation Model Results}

The discrete event simulation model was used for the BCS concept to examine the effect of factors and variances that are not easily accounted for by the other models. These were: varying the processing times, adding the effect of a periodic major inspection to the vehicle, accounting for weather and 
technical delays that result in vehicle rollbacks, accounting for end of service life, the effect of aborting to alternate landing sites, and the effect of catastrophic vehicle loss on the turnaround time and fleet size over the program life. For this illustration, the concept uses a processing facility in which all maintenance work is accomplished and then the payload is serially mated to the vehicle prior to moving to the launch pad. At the pad, the launch is accomplished or delayed based on a launch probability of $95 \%$. The other $5 \%$ were delayed either due to technical problems or weather based on Shuttle statistical distributions. Technical delays were assumed to cause a rollback prior to a new launch attempt. Weather delays were assumed to cause a 24 hour recycle time before another launch attempt. If delayed a second consecutive time, the vehicle is rolled back to the processing facility for rework before another launch is attempted. On launch and on orbit, aborts to alternate landing sites are probablistically determined. Return from these sites requires additional resources and time that affects the flight rate. After every 20 flights, the vehicle is required to undergo a major inspection, requiring more time and manpower than standard processing and impacting the flight rate. In addition, the service life for each vehicle is tracked and the vehicle replaced when its service life of 100 flights is reached. Catastrophic losses of $0.5 \%$ are assumed and accounted for along with the delays required to build a replacement vehicle.

The cumulative effect of each of these factors on the number of vehicles required over the program life is illustrated in Figure 10. Many additional factors which are not shown are impacted such as support facilities and crew requirements. The fleet size requirement of 3 as predicted by the R\&M model grows to 6 when these operating environment factors are considered. Over the life of the program, a total of 15

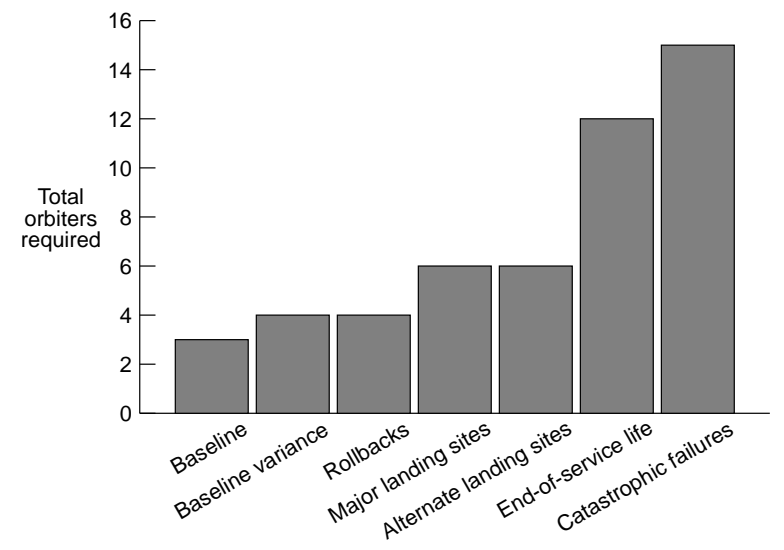

Figure 10. Cumulative effect of additional operational factors on total orbiters required over life cycle. vehicles will have to be built to maintain the fleet size to support the required flight rate.

\section{Discussion}

The purpose of these models is to provide insight into the effects of design and maintenance concept choices on the operations and support requirements of conceptual systems. They primarily provide guidance to the magnitude and direction of change that can be expected in time, manpower, resources and cost of decisions made during the conceptual phase of development. Since they are based on historical data they also provide a measure of how reasonable the estimates are relative to the experience of operational aircraft and launch vehicles. These do not preclude support beyond the bounds established by the historical data, but provide a basis for judging the credibility of these estimates. In general these models are expected value models and do not account for the variance that occurs in operational systems.

The R\&M model focuses on the maintenance and support of the launch vehicle up to launch. It does not address payload operations, launch or mission support although these can be accounted for with input from other sources. The model provides a means to combine data from diverse sources, Shuttle and aircraft, and from different time periods. It allows the user to make the comparisons in the same time frame and to account for the differences in growth rate of different technologies. The logistics model expands on this basic comparison to show the effects of design and support decisions on areas that are not as directly related to the design concept as in the R\&M model results. The simulation model is then used to enhance the results by accounting for effects of time and resource constraints over the program life.

From the results of the examples, it is obvious that maintenance policy is the major driver in defining the total maintenance burden for a system. The maintenance policy is not arbitrarily chosen and changed. The characteristics that have been captured by the parameters chosen to illustrate these policies represent the results of meeting the needs of different support environments. Changing the parameter values represents changes that have to correspond to changes in the requirements that set those policies in the first place. The model can only address the effects of 'what if' these changes could be made. Actual changes would have to identify the underlying requirements for these policies to effect real changes. 
Validation of these models is difficult because of lack of independent data. What information that is available has generally been used to develop the algorithms used in the models. The R\&M model was validated against independent aircraft parameters using data from a different time period. The results provided $\mathrm{R} \& \mathrm{M}$ parameters within $20 \%$ for 3 specific aircraft. The model could only be verified for the Shuttle data in a test case compared with the top level information that is known. The manpower requirements for Shuttle had to be inferred from system level data for each of the underlying subsystems. The distribution was based on a combination of the number of maintenance actions and the repair times for each subsystem. In general, cost information necessary to calibrate each of the logistic cost elements was not available and the model can only be used at this time to infer the effects of changes to the design or support environment. The logistics algorithms used were based on the experience and intuitive judgment of those who have worked the Shuttle and aircraft programs. They are not curve fit to empirical data. As with all simulation models a verification process needs to be performed on all code.

These models illustrate the potential benefits of defining support requirements during the conceptual design process. Unfortunately, they have of necessity been developed with less than the desired level of data from the Shuttle program. As this information becomes available, the models will be updated to provide results based on the most currently demonstrated capabilities. Operations and support analysis and estimations for future launch vehicles has always been somewhat of a subjective area. Through this process, the level of subjectivity can be reduced by providing results based on design, maintenance, and operating and support histories. These add validity to the results because they are traceable to demonstrated capability. These methods allow the user to define the support based on what can reasonably be achieved with current technologies and support policies. Only then can rationale judgment be made as to the potential improvement and value of introducing new technologies and support practices.

\section{Summary/Conclusions}

Methods have been presented which are under development for defining support requirements during the conceptual design phase. These analysis methods are based on comparability to support requirements for current operational aircraft and launch vehicles. The methods form a basis for providing relative support estimates for new launch vehicle designs and operating scenarios. The example presented illustrates how the models can be used to provide estimates that progressively expand the definition of the concept's support requirements. For the example used, the results show the maintenance support concept to be a larger driver of support requirements than improved $\mathrm{R} \& \mathrm{M}$ characteristics alone. Also, use of a simulation model provides a support definition that can account for the increased resources required in the operational environment that are not captured by expected value models. The relative changes to support requirements developed by these models can be used to help discriminate among new designs and support concepts.

\section{References}

1. Logistic Support Analysis, MIL-STD-1388, April 11, 1983.

2. Reliability-Centered Maintenance Requirements for Navel Aircraft, Weapons Systems and Support Equipment, MIL-STD-2173, January 26, 1986.

3. Failure Modes and Effects and Criticality Analysis, MILSTD-1629, November 24, 1980.

4. Schlagheck, R. A. and J. K. Byers. "Simulating The Operations of the Reusable Shuttle Space Vehicle." Proceedings of the 1971 Summer Computer Simulation Conference, pp. 192-152, July 1971.

5. Morris, W. D., T. A. Talay and D. G. Eide. "Operations Simulation for the Design of a Future Space Transportation System." Presented at the AIAA 21st Aerospace Sciences Meeting, paper no. 83-0140, January 1983.

6. Morris, W. D. and N. H. White. "A Space Transportation System Operations Model." NASA TM 100481, December, 1987.

7. Huseonica, W. F., private communication, "Shuttle II Data Base Development," Teledyne Brown Engineering, SC7490, Huntsville, AL, July 1987.

8. Ebeling, C. E., private communication, "The Determination of Operational and Support Requirements and Costs During The Conceptual Design of Space Systems," Grant No. NAG1-1-1327, University of Dayton, August, 1992. 
9. Ebeling, C. E., "Parametric Estimation of R\&M Parameters During the Conceptual Design of Space Vehicles." IEEE 1992 National Aerospace and Electronics Conference, Vol 3, Univ. of Dayton, Dayton OH, pp. 955-959.

10. Weber, T. F., "Reliability and Maintainability in Space Systems Design." Presented at the Aerospace Design Conference, Paper no. 93-1025, Feb 16-19, 1993.

11. Fleming, B. W., private communication, "Launch Vehicle Maintenance Analysis," Martin Marietta Manned Space Systems, NAS1-18230, Task 18, NASA Langley Research Center, Hampton, VA, November 1992.

12. Eshleman W., private communication, "Available Shuttle Systems Data Base Identification and Pathfinder Data Search" Lockheed Martin, NASA MSFC, Huntsville AL, May 31,1995.
13. Ehrlich, C. F., Jr., "Personnel Launch System (PLS) Study Final Report (DRD12)," NASA CR-187620, Oct.1991.

14. Cline, G. C., private communication, "Logistics Cost Analysis Model," Rockwell International Space Systems Division, NAS1-19243, Task 15, NASA Langley Research Center, Hampton, VA, October, 1994.

15. Morris, W. D., N. H. White and R. G. Caldwell. "HL-20 Operations and Support Requirements for the Personnel Launch System Mission," Journal of Spacecraft and Rockets, Vol. 30, No. 5, 1993, pp. 597-605.

16. Stanley, D. O., Engelund, W. C., Lepsch, R. A., McMillin, M.L., Wurster, K. E., Powell, R. W., Guinta, A. A., and Unal, R., "Rocket-Powered Single-Stage Vehicle Configuration Selection and Design," Journal of Spacecraft and Rockets, Vol. 31, No. 5, 1994, pp. 792798; also AIAA Paper 93-1053, Feb. 1993. 